\title{
Comparison of successive and simultaneous methods of pair presentation in paired-associate learning ${ }^{\prime}{ }^{2}$
}

\author{
Sam C. Brown, KANSAS STATE UNIVERSITY \\ Penny Brown, CARLETon COLLEGE
}

\begin{abstract}
Abstraet
Paired-associate learning was significantly retarded when pairs were presented simultaneously rather than successively for rehearsal. This difference was invariant with respect to degree of intralist similarity, size of rehearsal group, and whether pairs appeared in same or different rehearsal groups on each trial. Problem
\end{abstract}

Studies of paired-associate learning have typically involved the presentation of each individual pair alone in succession. Although a necessary feature of the anticipation procedure, the successive presentation of individual items has also characterized the alternative recall procedure (Battig \& Brackett, 1961). The latter technique, however, readily permits more than one pair to be presented simultaneously for study. Consequently, the present study was designed to compare the traditional successive method of pair presentation with a simultaneous method, wherein subsets of either two or four pairs are presented together for study. Since the relative efficiency of learning under the two methods may be expected to vary as a function of the consistency with which the same pairs are grouped together under the simultaneous method, as well as level of intralist similarity, variations in both of these factors were also incorporated into the present design.

\section{Method}

Nine main groups of 24 college-student Ss learned a 12-pair list of CVC's (from 75-85\% Archer (1960) association value) to a criterion of one errorless trial or a maximum of 25 trials. The groups differed with respect to (a) number of pairs (either 1, 2, or 4) presented together for simultaneous rehearsal on pairpresentation series of each trial, and (b) degree of formal intralist similarity. The low-, medium-, and $\mathrm{h}$ i g h-similarity lists varied with respect to both number and inter-item duplication of component letters, and were taken from Battig \& Brackett (1963). Half (8) of the Ss in each of the 2 and 4 conditions learned under Constant grouping, with the same pairs presented together on each trial, whereas the remainder learned under V a ried grouping, with unsystematic regrouping of pairs from trial to trial. Each of four different pair-grouping combinations was used for an equal number of 2- and 4-constant Ss, and the groups of pairs within each combination were arranged so as to yield comparable degrees of interpair similarity both within and between pair-groupings.

The pairs of each list were typed on $3 \times 5$ in index cards either individually (12 cards) or vertically in groups of two ( 6 cards) or four ( 3 cards) for presentation under the 1, 2, and 4 conditions, respectively. Each trial began with the successive presentation of the cards, through a manually-operated card-exposure device, at a rate of $3 \mathrm{sec}$. under the 1 condition, $6 \mathrm{sec}$. under the 2 condition, and $12 \mathrm{sec}$. under the 4 condition. Immediately following the pair-presentation series, all groups were shown successively, at a $3-\mathrm{sec}$. rate, each of the 12-stimulus terms alone in a different order, while $\mathrm{S}$ attempted to recall the correct response term paired with it.

All Ss were required to pronounce both members of each pair on a card only during pair-presentation series, and the order of pairs within cards under the 2 and 4 conditions, as well as the order in which the cards appeared, was unsystematically varied from trial to trial. The intertrial interval was approximately $10 \mathrm{sec}$.

\section{Results}

Table 1 presents the mean number of total errors for each condition, and for the Constant and Varied groups within the 2- and 4-grouping conditions. The substantial superiority of the 1 over the 2 and 4 conditions proved to be highly significant, $F(1,135)=26.89 ; p<.001$, as was also the increase in errors with increasing intralist similarity, $F(2,135)=25.58 ; p<.001$. However, there were no significant differences in errors between 2- and 4-grouping nor between Constant (113.8) and Varied (113.2) conditions, and no evidence of interactions among any of the variables (all $\mathrm{F}^{\prime} \mathrm{S}<1$ ).

In an effort to specify the source(s) of interference under the grouping conditions, the total number of Within-group substitution errors was tabulated separately for each $\mathrm{S}$ in the 2 and 4 conditions. A Within error was defined as the use of a response or stimulus term belonging to another pair contained in the same group on pair-presentation series. Since the pairs were regrouped on every trial under the Varied condition, the Within-error score for each Varied S was necessarTable 1. Mean Number of Total Errors for Groups and Conditions

\begin{tabular}{|c|c|c|c|c|c|}
\hline & \multicolumn{5}{|c|}{ Size of Group } \\
\hline $\begin{array}{c}\text { List } \\
\text { Similarity }\end{array}$ & $\begin{array}{l}\text { Sub- } \\
\text { Group }\end{array}$ & 4 & 2 & 1 & Total \\
\hline Low & $\begin{array}{l}\text { Constant } \\
\text { Varied }\end{array}$ & $\begin{array}{l}65.5 \\
78.4\end{array}$ & $\begin{array}{l}98.9 \\
79.3\end{array}$ & 53.8 & 71.6 \\
\hline Medium & $\begin{array}{l}\text { Constant } \\
\text { Varied }\end{array}$ & $\begin{array}{l}104.5 \\
107.6\end{array}$ & $\begin{array}{r}110.4 \\
98.5\end{array}$ & 43.3 & 84.6 \\
\hline High & $\begin{array}{l}\text { Constant } \\
\text { Varied }\end{array}$ & $\begin{array}{l}154.1 \\
154.1\end{array}$ & $\begin{array}{l}149.6 \\
161.4\end{array}$ & 108.2 & 139.3 \\
\hline & Total & 110.7 & 116.3 & 68.4 & \\
\hline
\end{tabular}


ily based upon only the actual pair-grouping used within each individual trial. Each of these scores was then converted to above-chance percentages by dividing the number of Within errors by the total number of overt substitution errors, and subtracting the proportion of Within errors expected by chance $(1 / 11$ and $3 / 11$ for the 2- and 4-grouping conditions, respectively). The results showed, as expected, significantly more percent (above chance) Within errors under the Constant (11.9) than under the Varied $(4.5)$ condition, $F(1,81)=4.00$; $\mathrm{p}<.05$, although both scores were significantly above chance expectancy, $F(1,40)=13.56 ; \mathrm{p}<.001$; F $(1,41)=5.72 ; p<.025$, respectively. All other main effects and interactions involving similarity, size of group, or constancy of grouping were not significant (all $\mathrm{F}^{\prime} \mathrm{s} \leq 1.33$ ). Additional analyses revealed a significant increase in percentage of overt errors (substitution plus extralist intrusions) from low (23.6) through medium (31.2) and high (44.2) similarity lists, $\mathrm{F}(2,135)=11.63 ; \mathrm{p}<.001$.

\section{Diseussion}

The observed inferiority of the present simultaneous method is indicative of an increase in interpair interference (Brown, 1964) under this as compared with the sučcessive method. Specifically, the contiguous presentation of groups of pairs may serve to enhance multiple associations among the stimulus and/or response members of these pairs, thereby leading to increased response competition at recall. The finding of significant above-chance Within errors both after repeated (Constant) and single (Varied) contiguous pair-presentations is consistent with such an interpretation, as well as with previous findings (Rotberg, 1964; Rotberg \& Woolman, 1963). Nevertheless, the failure to find a significant effect of either size of rehearsal group or of constancy suggests the existence of more complex intralist interactions and/or additional inhibitory mechanisms. One likely source of interference present under the simultaneous method was the change in procedure from simultaneous pair-presentation to individual pair testing at recall. Such a procedural change necessarily eliminated, particularly under the Constantgrouping condition, the pair-grouping context present during the pair-presentation series, thereby possibly reducing the number of stimulus cues available at recall.
The present detrimental effect of simultaneous rehearsal may be limited to tasks requiring the learning of specific S-R associations, and even here may not obtain if pairs are grouped together on the basis of some organizational principle, such as stimulus similarity (e.g., Rotberg, 1964; Rotberg \& Woolman, 1963). Moreover, factors responsible for interference within the present paired-associate task may serve to facilitate performance in different learning situations. For example, in the free-verbal recall of single items, response competition produced by grouping may improve retention by increasing item availability (e.g., Wickelgren, 1964).

The method of simultaneous pair-presentation as described here appears to be potentially useful not only for the investigation of intralist learning phenomena, but also for the specification and systematic manipulation of variables related to techniques of rehearsal, which heretofore have received relatively little experimental attention.

\section{Referenees}

ARCHER, E. J.A re-evaluation of the meaningfulness of all possible CVC trigrams. Psychol. Monogr., 1960, 74, No.10 (Whole No. 497).

BATTIG, W. F., \& BRACKETT, H. R. Comparison of anticipation and recall methods in paired-associate learning. Psychol. Rep., 1961, 9, 59-65.

BATTIG, W. F., \& BRACKETT, H. R. Transfer from verbaldiscrimination to paired-associate learning: II. Effects of intralist similarity, method, and percentage occurrence of response members. J. exp. Psychol., 1963, 65, 507-514.

BROWN, S. C. Interpair interference as a function of level of practice in paired-associate learning. J. exp. Psychol., 1964, $67,316-323$.

ROTBERG, I. C. Verbal paired-associate learning as a function of grouping similar stimuli or responses. J. exp. Psychol., 1964, $67,298-299$.

ROTBERG, I. C., \& WOLLMAN, M. Verbal paired-associate learning as a function of grouping similar stimuli or responses. J. exp. Psychol., 1963,65, 47-51.

WICKELGREN, W. A. Size of rehearsal group and short-term memory. J. exp. Psychol., 1964, 68, 413-419.

\section{Notes}

1. This research was supported by grant $\mathrm{MH}-10019$ from the National Institutes of Health and by a National Science Foundation Undergraduate Research Grant (GE-2931).

2. The authors wish to thank Marvin Bulgatz for his assistance and also William F. Battig and Robert C. Haygood for their valuable suggestions. 